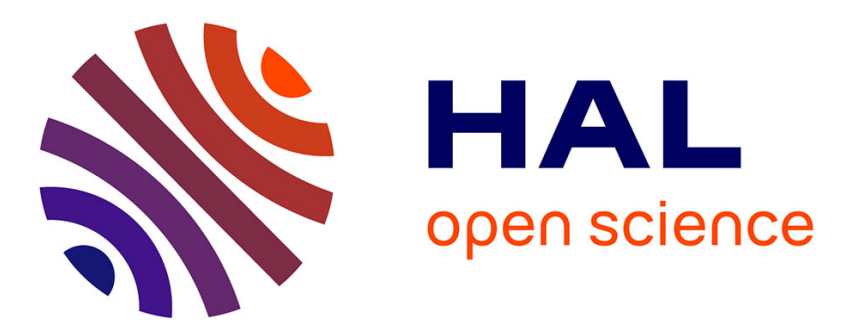

\title{
Editorial - Neurofeedback: a challenge for integrative clinical neurophysiological studies
}

\author{
Jean-Arthur Micoulaud Franchi, Camille Jeunet, Fabien Lotte
}

\section{To cite this version:}

Jean-Arthur Micoulaud Franchi, Camille Jeunet, Fabien Lotte. Editorial - Neurofeedback: a challenge for integrative clinical neurophysiological studies. Neurophysiologie Clinique $=$ Clinical Neurophysiology, 2020, 10.1016/j.neucli.2020.01.001 . hal-02436755

\section{HAL Id: hal-02436755 \\ https://hal.inria.fr/hal-02436755}

Submitted on 13 Jan 2020

HAL is a multi-disciplinary open access archive for the deposit and dissemination of scientific research documents, whether they are published or not. The documents may come from teaching and research institutions in France or abroad, or from public or private research centers.
L'archive ouverte pluridisciplinaire $\mathbf{H A L}$, est destinée au dépôt et à la diffusion de documents scientifiques de niveau recherche, publiés ou non, émanant des établissements d'enseignement et de recherche français ou étrangers, des laboratoires publics ou privés. 


\title{
EDITORIAL
}

\section{Neurofeedback: a challenge for integrative clinical neurophysiological studies}

\author{
Jean-Arthur MICOULAUD FRANCHI ${ }^{1 *}$, Camille JEUNET ${ }^{2}$, Fabien LOTTE $^{3}$
}

1 Service d'explorations fonctionnelles du système nerveux, clinique du sommeil, CHU de Bordeaux, place Amélie Raba-Léon, 33076 Bordeaux / USR CNRS 3413 SANPSY, université de Bordeaux, CHU Pellegrin, 33076 Bordeaux, France.

2 Laboratoire cognition, langues, langage, ergonomie (CLLE), CNRS / Université Toulouse JeanJaurès, 31058 Toulouse, France

3 Inria Bordeaux Sud-Ouest, Talence, France. LaBRI (CNRS / Univ. Bordeaux / Bordeaux INP), Talence, France.

* Corresponding author:

Dr. MICOULAUD FRANCHI Jean-Arthur

Services d'explorations fonctionnelles du système nerveux, Clinique du sommeil, CHU de Bordeaux, Place Amélie Raba-Leon, 33076 Bordeaux

E-mail adresse : jarthur.micoulaud@gmail.com 
In the article of Bismuth, Vialatte and Lefaucheur published in this issue of Neurophysiologie Clinique/Clinical Neurophysiology, the authors report the design of a single-center, single-blinded, randomized controlled study on neurofeedback [3]. Despite focusing on chronic neuropathic pain, the publication of this study protocol is a commendable initiative. Indeed, it highlights many of the important features that must be taken into account in order to inform the current debate on neurofeedback, through an integrative clinical neurophysiology standpoint.

Neurofeedback is a longstanding neurophysiological approach based on the fact that brain activity may be modulated by conditioning responses. This principle was first demonstrated through electroencephalographic (EEG) studies in the 1930s and 1940's. These studies investigated the EEG alpha blocking response [13], with the first study of Durup and Fessard at the Laboratoire de Physiologie des Sensations (Collége de France) [5], followed by the studies of Loomis in his laboratory at Tuxedo Park [14] and Jasper at the Montreal Neurological Institute (McGill University) [8]. The interest raised by these authors was not only in conditioning brain activity, but also in considering neurofeedback as a psychophysiological approach linking mental activities ("psycho-”) and brain activities (“-physiology”). As noted by Durup and Fessard in their seminal work on the conditioning of the EEG alpha blocking response: "l'attitude mentale du sujet intervient dans le déterminisme de la réaction d'arrêt" "15].

Subsequent studies in the 1960's confirmed that EEG alpha blocking could indeed be conditioned [12, 19] and that it was also possible to modulate brain activity through EEG and mental activities using neurofeedback, both in animals [26, 30] and humans [29]. This later finding paved the way for many therapeutic clinical applications dedicated to the reduction of mental symptoms and cognitive impairments induced by brain pathologies (for a review see [18]). These findings also form the basis of developments focussing on the reduction of functional limitations through rehabilitative procedures based on Brain-Computer Interface (BCI) technologies (for a review see [4]). Neurofeedback has thus attracted the attention of the clinical neurophysiological community [1, 2], Nonetheless, a debate regarding the mechanisms through which neurofeedback may benefit therapeutic procedures was initiated in the Lancet Psychiatry [17, 21, 27, 28, 34, 36] and spread to Brain [6, 23-25, 31, 32, 38], the Journal of Attention Disorders [20, 37], and American Psychologist [16, 33, 35]. In summary, the question is the following: does neurofeedback operate through a specific neurophysiological effect on the modulation of the targeted brain activity [16]? Indeed, many other mechanisms could be at play: patient's perceptions of self-efficacy, social reinforcement, or a general but non-specific cognitive training related to the environment of a neurofeedback session [16].

Such mechanisms most likely play a role in neurofeedback efficacy, insofar as a few recent studies did not find any superior effect of neurofeedback over sham neurofeedback (based on signal unrelated to the targeted brain activity) [25, 28]. Thus, other well-designed studies for neurofeedback are

\footnotetext{
${ }^{1}$ An English translation would be: "the subject's mental attitude contributes to the occurrence of the blocking response"
} 
urgently needed: i) double blind randomized controlled trials (RCT) with consensual standards of reporting, but also ii) clinical neurophysiological relevance of the mechanism through which neurofeedback might have a therapeutic benefit $[6,16]$.

Concerning RCT, a recent checklist has been published in Brain [22]. The "Consensus on the Reporting and Experimental Design of clinical and cognitive-behavioural Neurofeedback studies” (CRED-nf) best practices checklist 2019 is intended to "encourage robust experimental design and clear reporting for clinical and cognitive-behavioural neurofeedback experiments" as a complement to the Consolidated Standards of Reporting Trials (CONSORT) guidelines. Clinical neurophysiologists can only encourage such an initiative, which could help to disentangle the different mechanisms underlying clinical efficacy of neurofeedback. Interestingly enough, the article of Bismuth, Vialatte and Lefaucheur satisfies almost all the design and reporting criteria from the CRED-nf checklist, which is not so common and thus worth noting. Only one reporting criteria appears to be missing: how the feedback was precisely designed and provided (e.g., information on the reward threshold, reward amount and frequency or the EEG-to-feedback mapping). Including such information would have made the protocol reporting even more commendable.

Nevertheless, concerning clinical neurophysiological relevance, we think that the article of Bismuth, Vialatte and Lefaucheur published in this issue helps to go a step further [3], and could be used in the iteration process of the improvement of the CRED-nf checklist. Indeed, all clinical interventions need a model (or theory) of change relevant to the field. Thus, design of neurofeedback protocols should be conducted in accordance with a model of change to demonstrate the causal connection between the constructs of the model and the observed clinical and neurophysiological changes.

For doing so, the first need for neurofeedback is to target a brain activity that is based on a relevant psychophysiological model. By psychophysiologically relevant, we mean that there should be scientific evidence of a clear relationship between the particular neurophysiological marker targeted by the neurofeedback and the cognitive process being studied. A recent "EEG-Copeia" for neurofeedback, such as the "Pharmacopeia" for psychopharmacology has been proposed [15]. An "EEG-Copeia" has been defined as "an organized list of scientifically validated EEG markers, characterized by a specific association with an identified cognitive process, that define a psychophysiological unit of analysis useful for mental or brain disorder evaluation and treatment” [15]. We think that the article of Bismuth, Vialatte and Lefaucheur is an emblematic example of this psychophysiological approach in the choice of the EEG target to relieve chronic neuropathic pain in patients with painful peripheral neuropathy.

The second need for neurofeedback is to base evaluation of the psychosocial factors and mental strategies (Items 3a, 3b, 3c of the CRED-nf [22]) on an explicit learning model during neurofeedback. A recent review of the different learning models in behavioral, developmental and cognitive psychology of the feedback learning processes has been proposed [7]. Despite the fact that there is no consensus on the best learning model for neurofeedback, neurofeedback protocols should 
attempt to investigate the variables that could be relevant from a clinical neurophysiological standpoint within an integrated model. Two recent integrated models have been proposed: the psychoengineering model [7] and a cognitive model inspired by BCI research [10], which could be very useful for the field of neurofeedback research [9, 11]. We think that the choice of metric of psychosocial factors and mental strategies in the article of Bismuth, Vialatte and Lefaucheur is also an emblematic example of evaluation grounded in a learning model that enables us to rigorously consider the interests and challenges but also the perils and pitfalls of neurofeedback. Indeed, since the claim of Durup and Fessard, this encourages development of integrative clinical neurophysiology approaches to face "l'attitude mentale du sujet / the subject's mental attitude" in a study protocol.

\section{Conflict of interest}

The authors declare that there is no conflict of interest regarding the publication of this article.

\section{References}

[1] Arns M, Batail JM, Bioulac S, Congedo M, Daudet C, Drapier D, et al. Neurofeedback: One of today's techniques in psychiatry? Encephale 2017; 43:135-45.

[2] Batail JM, Bioulac S, Cabestaing F, Daudet C, Drapier D, Fouillen M, et al. EEG neurofeedback research: A fertile ground for psychiatry? Encephale 2019; 45:245-55.

[3] Bismuth J, Vialatte F, Lefaucheur JP. Relieving peripheral neuropathic pain by increasing the power-ratio of low- $\beta$ over high- $\beta$ activities in the central cortical region with EEG-based neurofeedback: study protocol for a controlled pilot trial (SMRPain study). Neurophysiol Clin 2020; in press.

[4] Daly JJ, Wolpaw JR. Brain-computer interfaces in neurological rehabilitation. Lancet Neurol 2008;7:1032-43.

[5] Durup G, Fessard A. L'électroencéphalogramme de l'homme. Observations psychophysiologiques relatives à l'action des stimuli visuels et auditifs. L'Année Psychologique 1935;36:1-32.

[6] Fovet T, Micoulaud-Franchi JA, Vialatte FB, Lotte F, Daudet C, Batail JM, et al. On assessing neurofeedback effects: should double-blind replace neurophysiological mechanisms? Brain 2017;140:e63.

[7] Gaume A, Vialatte A, Mora-Sánchez A, Ramdani C, Vialatte F. A psychoengineering paradigm for the neurocognitive mechanisms of biofeedback and neurofeedback. Neurosci Biobehav Rev 2016;68:891-910.

[8] Jasper H, Shagass C. Conditionning of the occipital alpha rythm in man. J Exp Psychol 1941;28:373-88.

[9] Jeunet C, Glize B, McGonigal A, Batail JM, Micoulaud-Franchi JA. Using EEG-based brain computer interface and neurofeedback targeting sensorimotor rhythms to improve motor skills: Theoretical background, applications and prospects. Neurophysiol Clin 2019;49:12536.

[10] Jeunet C, K’Naoua B, Lotte F. Towards a cognitive model of MI-BCI tasks. International BCI Conference (Graz) 2017.

[11] Jeunet C, Lotte F, Batail JM, Philip P, Micoulaud Franchi JA. Using Recent BCI Literature to Deepen our Understanding of Clinical Neurofeedback: A Short Review. Neuroscience 2018;378:225-33. 
[12] Kamiya J. Biofeedback training in voluntary control of EEG alpha rhythms. Calif Med 1971;115:44.

[13] Kamiya J. The First Communications About Operant Conditioning of the EEG. J Neurother 2011;15:65-73.

[14] Loomis A, Harvey E, Hobart G. Electrical potentials of the human brain. J Exp Psychol 1936;19:249-79.

[15] Micoulaud-Franchi JA, Batail JM, Fovet T, Philip P, Cermolacce M, Jaumard-Hakoun A, et al. Towards a Pragmatic Approach to a Psychophysiological Unit of Analysis for Mental and Brain Disorders: An EEG-Copeia for Neurofeedback. Appl Psychophysiol Biofeedback 2019;44:151-72.

[16] Micoulaud-Franchi JA, Fovet T. A framework for disentangling the hyperbolic truth of neurofeedback: Comment on Thibault and Raz (2017). Am Psychol 2018;73:933-5.

[17] Micoulaud-Franchi JA, Fovet T. Neurofeedback: time needed for a promising nonpharmacological therapeutic method. Lancet Psychiatry 2016;3:e16.

[18] Micoulaud-Franchi JA, McGonigal A, Lopez R, Daudet C, Kotwas I, Bartolomei F. Electroencephalographic neurofeedback: Level of evidence in mental and brain disorders and suggestions for good clinical practice. Neurophysiol Clin 2015;45:423-33.

[19] Milstein V. Contingent Alpha Blocking: Conditioning or Sensitization? Electroencephalogr Clin Neurophysiol 1965;18:272-7.

[20] Pigott HE, Cannon R, Trullinger M. The Fallacy of Sham-Controlled Neurofeedback Trials: A Reply to Thibault and Colleagues (2018). J Atten Disord 2018:1087054718790802.

[21] Pigott HE, Trullinger M, Harbin H, Cammack J, Harbin F, Cannon R. Confusion regarding operant conditioning of the EEG. Lancet Psychiatry 2017;4:897.

[22] Ros T, Enriquez-Geppert S, Zotev V, Young K, Wood, G., Whitfield-Gabrieli S, et al. Consensus on the reporting and experimental design of clinical and cognitive-behavioural neurofeedback studies (CRED-nf checklist). Brain 2020; in press;

https://doi.org/10.31234/osf.io/nyx84.

[23] Schabus M. Reply: Noisy but not placebo: defining metrics for effects of neurofeedback. Brain 2018;141:e41.

[24] Schabus M. Reply: On assessing neurofeedback effects: should double-blind replace neurophysiological mechanisms? Brain 2017;140:e64.

[25] Schabus M, Griessenberger H, Gnjezda MT, Heib DPJ, Wislowska M, Hoedlmoser K. Better than sham? A double-blind placebo-controlled neurofeedback study in primary insomnia. Brain 2017;140:1041-52.

[26] Schafer RJ, Moore T. Selective attention from voluntary control of neurons in prefrontal cortex. Science 2011;332:1568-71.

[27] Schonenberg M, Wiedemann E, Schneidt A, Scheeff J, Logemann A, Keune PM, et al. Confusion regarding operant conditioning of the EEG - Authors' reply. Lancet Psychiatry 2017;4:897-8.

[28] Schonenberg M, Wiedemann E, Schneidt A, Scheeff J, Logemann A, Keune PM, et al. Neurofeedback, sham neurofeedback, and cognitive-behavioural group therapy in adults with attention-deficit hyperactivity disorder: a triple-blind, randomised, controlled trial. Lancet Psychiatry 2017;4:673-84.

[29] Sitaram R, Ros T, Stoeckel L, Haller S, Scharnowski F, Lewis-Peacock J, et al. Closed-loop brain training: the science of neurofeedback. Nat Rev Neurosci 2016;18:86-100.

[30] Sterman MB, Howe RC, Macdonald LR. Facilitation of spindle-burst sleep by conditioning of electroencephalographic activity while awake. Science 1970;167:1146-8.

[31] Thibault RT, Lifshitz M, Raz A. The climate of neurofeedback: scientific rigour and the perils of ideology. Brain 2018;141:e11.

[32] Thibault RT, Lifshitz M, Raz A. Neurofeedback or neuroplacebo? Brain 2017;140:862-4.

[33] Thibault RT, Raz A. A consensus framework for neurofeedback research (and the perils of unfounded neuroreductionism): Reply to Micoulaud-Franchi and Fovet (2018). Am Psychol 2018;73:936-7.

[34] Thibault RT, Raz A. Neurofeedback: the power of psychosocial therapeutics. Lancet Psychiatry 2016;3:e18. 
[35] Thibault RT, Raz A. The psychology of neurofeedback: Clinical intervention even if applied placebo. Am Psychol 2017;72:679-88.

[36] Thibault RT, Raz A. When can neurofeedback join the clinical armamentarium? Lancet Psychiatry 2016;3:497-8.

[37] Thibault RT, Veissiere S, Olson JA, Raz A. Treating ADHD With Suggestion: Neurofeedback and Placebo Therapeutics. J Atten Disord 2018;22:707-11.

[38] Witte M, Kober SE, Wood G. Noisy but not placebo: defining metrics for effects of neurofeedback. Brain 2018;141:e40. 